\title{
Disease awareness in patients with COPD: measurement and extent
}

This article was published in the following Dove Press journal:

International Journal of COPD

Ilaria Baiardini,' Paola

Rogliani, ${ }^{2}$ Pierachille Santus, ${ }^{3}$

Angelo G Corsico, ${ }^{4}$ Marco

Contoli, ${ }^{5}$ Nicola Scichilone, 6

Fabiano Di Marco, ${ }^{7}$ Patrizia

Lessi, ${ }^{8}$ Carla Scognamillo, ${ }^{8}$

Giorgia Molinengo, ${ }^{9}$ Fabio

Ferri, ${ }^{10}$ Vincenzo Patella, ${ }^{\prime}$

Giuseppe Fiorentino, ${ }^{12}$ Mauro

Carone, ${ }^{13}$ Fulvio Braido ${ }^{14}$

'Department of Biomedical Sciences, Humanitas University, Milan, Italy;

${ }^{2}$ Respiratory Unit, Department of

Experimental Medicine, University of Rome

"Tor Vergata", Rome, Italy; ${ }^{3}$ Department

of Biomedical and Clinical Sciences

(DIBIC), University of Milan, Milan, Italy;

${ }^{4}$ Department of Internal Medicine and

Therapeutics, University of Pavia, Pavia,

Italy; ${ }^{5}$ Department of Medical Sciences,

University of Ferrara, Ferrara, Italy;

${ }^{6}$ Department of Biomedicine and Internal

and Specialistic Medicine (DIBIMIS),

University of Palermo, Palermo, Italy;

${ }^{7}$ Department of Health Sciences, University

of Milan, Milan, Italy; ${ }^{8}$ Boehringer Ingelheim,

Milan, Italy; ${ }^{9}$ Department of Psychology,

University of Turin, Turin, Italy; ${ }^{10}$ MediNeos

Observational Research, Modena, Italy;

"U.O. di Allergologia e Immunologia

Clinica del Dipartimento delle Discipline

Mediche, Battipaglia, Italy; ${ }^{12}$ U.O.C.

Malattie, Fisiopatologia e Riabilitazione

Respiratoria A.O. dei Colli, Naples, Italy;

${ }^{13}$ UOC Pneumologia, ICS Maugeri, IRCCS

Cassano Murge, Murge, Italy; ${ }^{14}$ Department of Internal Medicine, Respiratory Diseases and Allergy Clinic, University of Genova,

Azienda Policlinico IRCCS San Martino,

Genoa, Italy

Correspondence: Paola Rogliani Respiratory Unit, Department of Experimental Medicine. University of Rome "Tor Vergata," Via Montpellier I, 00I33 Rome, Italy

Tel +39620903616

Email paola.rogliani@uniroma2.it
Background: Patient awareness of COPD refers to knowledge and acceptance of the disease and its treatment. Although it is relevant to management and outcomes, the disease awareness of patients is poorly investigated, and no validated questionnaires are currently available. We aimed to develop the novel Disease Awareness in COPD Questionnaire (DACQ), which was validated in relation to demographic and clinical features, in patients participating in the SATisfaction and Adherence to COPD Treatment (SAT) study.

Methods: DACQ was developed according to a list of items regarding the patient's knowledge, acceptance, and perception of COPD as well as of treatment needs. The questionnaire was validated by assessing internal structure and consistency, correlations with other patient-reported outcomes, and stability over time. Furthermore, the extent of disease awareness of patients enrolled in the SAT study was assessed by using DACQ, and correlations with demographic and clinical features were evaluated.

Results: DACQ was composed of four domains. Overall reliability and stability over time were adequate; correlations between DACQ and other tools measuring different constructs (ie, treatment satisfaction, illness perception, impact of COPD symptoms on daily life, and dyspnea severity) were, as expected, more limited. In the enrolled patient sample, a suboptimal level of disease awareness $(<70 \%)$ was detected, especially in terms of disease acceptance and perception. Disease knowledge was positively associated with COPD severity, while the impact of symptoms on daily life was negatively associated with disease acceptance, awareness of treatment needs, and overall awareness.

Conclusion: DACQ proved to be a reliable tool to assess awareness in COPD patients. Awareness of COPD patients need to be improved.

Clinical trial registration: ClinicalTrials.gov ID\# NCT02689492.

Keywords: COPD, awareness, questionnaire, patient satisfaction

\section{Plain language summary}

The more patients with COPD are aware of the disease, treatment, and management, the more likely is their engagement, resulting in a better COPD care. Although relevant to disease management and outcome, disease awareness is poorly investigated, and no validated questionnaires considering all its domains are currently available to measure its extent.

We developed the Disease Awareness in COPD Questionnaire (DACQ), which was validated in the patients with COPD enrolled in the observational, multicentre, prospective "SATisfaction and Adherence to COPD Treatment" (SAT) study.

The validated DACQ proved to be a reliable patient-reported outcome tool for measuring the complex and multifaceted disease awareness of patients with COPD.

A well-known problem in COPD management is the low rate of adherence to long-term treatment. Assessment and reinforcement of awareness may be helpful for improving this issue. submit your manuscript | www.dovepress.com

Dovepress if in 1
International Journal of COPD 2019:14 I-II

(c) (i) (c) 2019 Baiardini et al. This work is published and licensed by Dove Medical Press Limited. The full terms of this license are available at https://www.dovepress.com/terms.php cc. hereby accept the Terms. Non-commercial uses of the work are permitted without any further permission from Dove Medical Press Limited, provided the work is properly attributed. For permission for commercial use of this work, please see paragraphs 4.2 and 5 of our Terms (https://www.dovepress.com/terms.php).
s. 


\section{Introduction}

COPD is the third leading cause of death worldwide ${ }^{1}$ and a cause of patients' disability. ${ }^{2,3}$ Disease awareness has been defined as understanding the disease and being conscious of it, its prognosis, and associated life expectations, as originated from the personal rework of information with which the patient has been provided. ${ }^{4}$ While patient education is essential for a successful management of a disorder, the concept of awareness can be extended beyond mere information and knowledge on the diagnosis and features of the disease. It should include all domains establishing a full awareness of the disease, its consequences, and the burden of the condition, as well as the understanding of the need for a therapeutic regimen. Programs mainly aimed at updating knowledge, according to the GOLD, ${ }^{5}$ and at enhancing the consciousness of COPD, its management, and importance as a public health problem and are being implemented in several countries. ${ }^{6}$ The National Heart, Lung, and Blood Institute has taken several initiatives since 2007 to raise the awareness of patients and their families in relation to specific health topics, including asthma ${ }^{7}$ and $\mathrm{COPD}^{8}$ in cooperation with leading professional societies, health organizations, and advocacy groups. ${ }^{9,10}$ Unsatisfying adherence to treatment is a well-known problem in COPD management, despite the huge disease burden. Indeed, at least half of patients receiving long-term pharmacotherapy do not adhere to treatment. ${ }^{11-14}$

The more patients are aware of the disease, treatment, and management, the more likely is their active collaboration, resulting in better COPD care. Therefore, further efforts are needed to investigate all components of disease awareness and to develop targeted training and suitable educational initiatives.

Literature data on awareness of COPD are still poor and limited only to some components of the paradigm, such as patient's information, beliefs, perceptions, and perspectives on treatments. ${ }^{15}$ The level of knowledge and understanding about the disease have been explored in the general population, ${ }^{15,37}$ in current smokers, ${ }^{10,38}$ and in COPD patients ${ }^{9}$ by means of surveys or unvalidated questionnaires. Unfortunately, no validated tools are currently available for measuring different dimensions of patients' COPD awareness.

The aim of this study was to develop and validate the novel Disease Awareness in COPD Questionnaire (DACQ) in patients participating in the SATisfaction and adherence to COPD treatment (SAT) study (SAT; ClinicalTrials.gov ID\# NCT02689492). The purpose was to obtain a short, simple, and user-friendly tool that can be used both in research and in clinical practice. The extent of patients'
COPD awareness was assessed by using the DACQ tool, and its correlations with disease features and patients' outcomes were examined.

\section{Methods SAT study design}

The SAT study patients were required to be $\geq 40$ years of age, receiving stable pharmacological COPD treatment, and have had no exacerbations for at least 3 months. They were enrolled in 20 Italian pulmonological centers and followed up to 12 months. Follow-up visits occurred every 6 months (with 1 month of tolerance). The SAT observational study was conducted in accordance with the amended Declaration of Helsinki. The University of Genoa Ethic Committee (Protocol No: 343REG2015) approved the study protocol, and written informed consent was obtained from all patients. The institutional ethics committees involved in the study are listed in Table S1. No individual data will be shared. Further data will be published with main clinical trial results and other subanalysis (eg, pharmacoeconomic data); the data will be shared through open access manuscripts.

At the enrollment visit and at 6-month follow-up, patients were administered the list of items approved by the Steering Committee along with the Brief-Illness Perception Questionnaire (B-IPQ), ${ }^{16}$ the COPD Assessment Test (CAT), ${ }^{17}$ the Treatment Satisfaction Questionnaire for Medication (TSQM-9), ${ }^{18,19}$ and the modified Medical Research Council (mMRC) dyspnea scale. ${ }^{20}$

\section{DACQ development and validation}

A list of 38 items exploring patient's knowledge, acceptance, and perception of COPD and awareness of treatment needs was generated by physicians and experts in health psychology, based on patients' input and literature search. Applying two rounds of the Delphi method, ${ }^{21}$ the SAT study Steering Committee members identified the most relevant items to characterize COPD awareness, suggested additional items, and rephrased the existing ones, if appropriate.

At study inclusion, SAT study patients were asked to complete the questionnaire with the selected items. The DACQ total and domain scores were computed as the sum of nonmissing items, after having assigned the appropriate score to each item in such a way that higher scores reflected a higher grade of awareness. Scores were normalized to a scale ranging from 0 to 100 .

An exploratory factor analysis was conducted to identify potential domains, while Rasch models for polytomous items ${ }^{22-26}$ were used (according to the Item Response Theory 
approach) to evaluate the scalability of items and to test whether patients used the items response scale correctly. ${ }^{27,28}$ For the Rasch analysis, information-weighted (INFIT) and outlier-sensitive (OUTFIT) indices were considered. Moreover, item-person maps, which provide both the person measures and the item measures on the same linear (logit) scale, were plotted to determine how items were suited to the patient's ability. Items with absolute factor loadings $<0.3$ in the factor analysis, or not fitting the Rasch model, were removed.

The scale homogeneity was evaluated by means of the Cronbach's coefficient $\alpha$. Less correlated items were removed, and the analysis was repeated until an acceptable level of reliability ( $\alpha \geq 0.70)$ was achieved. ${ }^{29,30}$

Internal consistency, construct validity, and stability after 6 months were evaluated as psychometric characteristics of the final DACQ by means of Cronbach's $\alpha$, Spearman correlation coefficient $\rho_{\mathrm{S}}$ with other questionnaires and scales used in the study and intraclass correlation coefficient, ${ }^{31}$ respectively.

\section{Disease awareness extent}

The levels of awareness, as well as the distribution of the DACQ total and domain scores with respect to the demographic and clinical characteristics, were evaluated at study entry.

Two-sample $t$-test and ANOVA (or respective analogous nonparametric tests, if appropriate) were used to evaluate the statistical significance of differences in scores among different groups of patients. Moreover, the associations between awareness and patient age, gender, COPD severity (based on the GOLD 2017 risk categories), ${ }^{5}$ and impact of symptoms (by means of the CAT score) were investigated with a multivariable linear regression analysis.

Analyses were performed using SAS 9.4, SPSS v. 20.0, and WINSTEPS 3.72.3. Statistical analyses, project management, and quality control were performed by MediNeos Observational Research (Modena, Italy).

\section{Results \\ DACQ development and validation}

Starting from the initial list of items, the Delphi method led to a provisional questionnaire of 27 items. Patients enrolled in the SAT study were administered the 27-item questionnaire; subject demographic and clinical characteristics are reported in Table 1. At the enrollment visit, the frequency of missing answers to each item was $<3 \%$, which is below
Table I Demographic and clinical characteristics of patients at the enrollment visit

\begin{tabular}{|c|c|}
\hline Total number of patients & 401 \\
\hline Age (years); mean $\pm S D$ & $71.7 \pm 7.6$ \\
\hline \multicolumn{2}{|l|}{ Gender, N (\%) } \\
\hline Males & $299(74.6)$ \\
\hline Females & $102(25.4)$ \\
\hline \multicolumn{2}{|l|}{ Smoking habits, N (\%) } \\
\hline Nonsmokers & $21(5.2)$ \\
\hline Former smokers & $282(70.3)$ \\
\hline Current smokers & $98(24.5)$ \\
\hline \multicolumn{2}{|l|}{ Highest education level, $N(\%)^{a}$} \\
\hline None & $6(1.9)$ \\
\hline Primary school & $138(42.7)$ \\
\hline Middle school & $105(32.5)$ \\
\hline High school & $5 I(I 5.8)$ \\
\hline Academic degree & $23(7.1)$ \\
\hline COPD duration (years); median & $4.9(2.1-9.2)$ \\
\hline (25th-75th percentile) $(\mathrm{N}=40 \mathrm{I})$ & \\
\hline CAT score; mean $\pm \mathrm{SD}(\mathrm{N}=40 \mathrm{I})$ & $15.7 \pm 7.8$ \\
\hline \multicolumn{2}{|l|}{ CAT score group, $\mathrm{N}(\%)$} \\
\hline CAT $<10$ & $96(23.9)$ \\
\hline $\mathrm{CAT} \geq 10$ & $305(76.1)$ \\
\hline \multicolumn{2}{|l|}{ COPD GOLD 2017 grade, $N(\%)^{a}$} \\
\hline Grade A & $70(19.1)$ \\
\hline Grade B & $254(69.4)$ \\
\hline Grade C & $2(0.6)$ \\
\hline Grade D & $40(10.9)$ \\
\hline FEV , predicted (\%); mean \pm SD $(N=369)$ & $64.7 \pm 28.7$ \\
\hline
\end{tabular}

Note: aPatients with missing information were not considered.

Abbreviations: CAT, COPD Assessment Test; $\mathrm{FEV}_{1}$, forced expiratory volume in one second.

the $5 \%$ recommended threshold. ${ }^{32}$ Therefore, the issue of missing data was not further dealt with.

Factor and Rasch analyses were conducted on the 347 structured questionnaires that were completed by patients at study entry. Four distinct latent factors with eigenvalues $>1$ were extracted: items $7,15,16,22,24$, and 25 were allocated to the first factor; items $3,4,9,12,14,17,18$, and 23 to the second one; items 1, 2, 19, and 26 to the third one; and items 5, 8,10,11,13,20,21, and 27 to the fourth one (Table S2). On the basis of the question text of the items that such factors were composed of, it was deduced that these four domains assess the patients' Disease acceptance, Awareness of treatment needs, Disease knowledge, and Disease perception, respectively. Therefore, COPD awareness was conceptualized as a composite, aggregate construct of these four domains, ${ }^{39}$ meaning that such domains combine to produce the latent construct. Moreover, on the basis of the factor analysis results (Table S2), only item 20 (assigned to the fourth factor) might have been allocated to other domains. We did not identify evidence of other possible relevant overlap among the four extracted factors. 
This also supports the aggregate structure of the DACQ latent construct.

The item location and the fit statistics resulting from the Rasch analysis are reported in Table S3: all the INFIT and OUTFIT values were satisfactory, with the only exceptions being items 6 and 25 .

According to the findings of factor and Rasch analyses, and based on the clinical interpretation of preliminary results as well, five items $(3,6,11,16$, and 17) were removed, resulting in a 22 -item version of the questionnaire.

To evaluate the shortened scale homogeneity, 339 patients at enrollment also completed the B-IPQ, mMRC, TSQM-9, and CAT questionnaires and scales and were considered. At first, the overall evaluation of the 22-item questionnaire resulted in a Cronbach's $\alpha$ coefficient of 0.69 . The removal of the least correlated items (ie, items 13 and 27, which also showed a nonsatisfactory performance in the factor and Rasch model analyses) led to an overall $\alpha=0.72$.

No further selection of the items was deemed to be necessary, leading to a final pool of 20 items (Table 2). The English translation of selected items (not linguistically validated) is reported in Table 2 only for informative purposes.
Both the global DACQ and its four domains showed an acceptable-to-good internal consistency (Cronbach's $\alpha$ coefficients were 0.81 for disease acceptance, 0.73 for awareness of treatment needs, 0.70 for disease knowledge, and 0.56 for disease perception). Regarding construct validity, the correlation coefficients between the DACQ score and the TSQM-9 global satisfaction score $\left(\rho_{\mathrm{S}}=0.40\right)$, B-IPQ $\left(\rho_{\mathrm{S}}=-0.40\right), \operatorname{CAT}\left(\rho_{\mathrm{S}}=-0.29\right)$, and $\operatorname{mMRC}\left(\rho_{\mathrm{S}}=-0.19\right)$ were calculated (all $P \leq 0.0001$ ). All correlations were in the expected direction, but the magnitude of association was limited, meaning that, as expected, the DACQ construct is correlated with but different from those measured by the other considered patient-reported outcomes (PROs; ie, treatment satisfaction, illness perception, impact of COPD symptoms on daily life, and dyspnea grade).

The stability over time of the DACQ was evaluated after 6 months on 79 patients with stable disease (CAT score variation $\leqq 1$ point). Almost all items showed a moderateto-good stability, ${ }^{33}$ with intraclass correlation coefficient values ranging from 0.33 to 0.64 (Table S4). The stability of the DACQ total and domains scores was satisfactory ( $\rho_{S}=0.65$ for disease acceptance, $\rho_{S}=0.60$ for awareness of

Table 2 Structure of the Disease Awareness in COPD Questionnaire (DACQ)

\begin{tabular}{|c|c|c|}
\hline Item \# & Item text & Domain \\
\hline 01 & I need to be treated with inhaled drugs forever & Disease knowledge \\
\hline 02 & I believe that COPD tends to worsen over time & Disease knowledge \\
\hline 04 & A regular administration of drugs allows me to move with less effort & Awareness of treatment needs \\
\hline 05 & I think that COPD is a mild disease since it is treated with inhaled drugs & Disease perception \\
\hline 07 & I cannot easily accept to have COPD & Disease acceptance \\
\hline 08 & Inhaled drugs are less effective than pills, tablets, or other oral medications & Disease perception \\
\hline 09 & Treatments for COPD must be taken regularly & Awareness of treatment needs \\
\hline 10 & I prefer to ignore COPD symptoms & Disease perception \\
\hline 12 & A regular therapy reduces the risk of sudden worsening & Awareness of treatment needs \\
\hline 14 & Inhaled drugs help improving the fatigue in breathing & Awareness of treatment needs \\
\hline 15 & I am worried about my COPD & Disease acceptance \\
\hline 18 & Spirometry is a fundamental examination for the COPD diagnosis & Awareness of treatment needs \\
\hline 19 & I will never heal from my COPD & Disease knowledge \\
\hline 20 & I think I will never heal from my COPD because there is lack of effective drugs & Disease perception \\
\hline 21 & I prefer not to think about my COPD & Disease perception \\
\hline 22 & I am angry because of my COPD & Disease acceptance \\
\hline 23 & Drugs for COPD improve my symptoms & Awareness of treatment needs \\
\hline 24 & I am having difficulty living with COPD symptoms & Disease acceptance \\
\hline 25 & I wonder why COPD happened to me & Disease acceptance \\
\hline 26 & COPD is a chronic disease and it is not possible to heal from it & Disease knowledge \\
\hline
\end{tabular}

Notes: Final list of the 20 selected items and of the respective domains. Seven items that were removed during the selection process are not shown. The questionnaire with the indicated items was administered to patients in their original, Italian version. The English translation provided in the table was not linguistically validated, and it is shown only for informative purpose. Items \#03, \#06, \#II, \#I3, \#I6, \#I7, and \# 27 were removed from the final DACQ, based on the results of the validation statistical tests performed. 


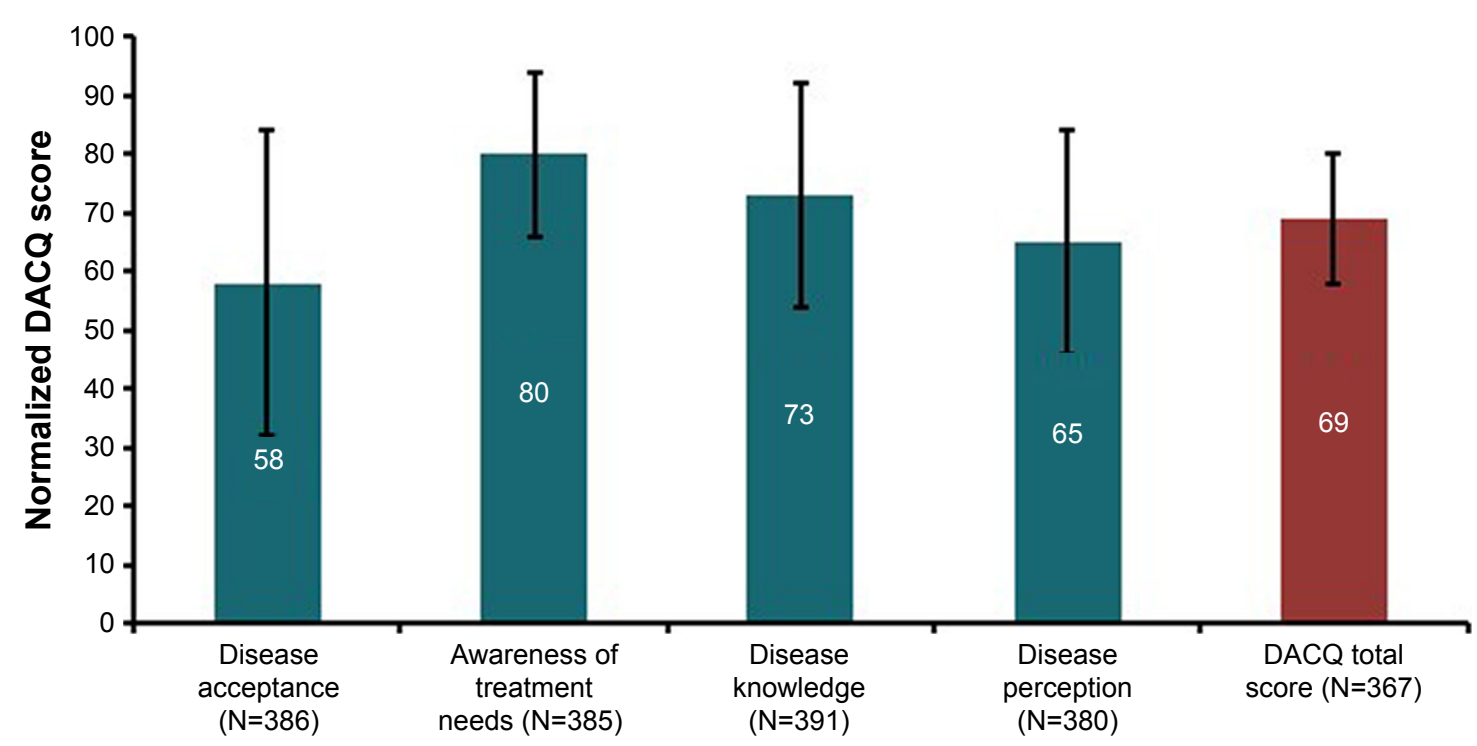

Figure I Patients' COPD awareness at enrollment.

Notes: Normalized DACQ scores (range 0-100) of COPD patients at enrollment visit; higher scores represent higher levels of awareness; mean values (bars), I SD (whiskers). Abbreviation: DACQ, Disease Awareness in COPD Questionnaire.

treatment needs, $\rho_{\mathrm{S}}=0.61$ for disease knowledge, $\rho_{\mathrm{S}}=0.38$ for disease perception, and $\rho_{\mathrm{s}}=0.54$ for DACQ total score; all $P<0.001)$.

\section{Disease awareness extent}

The following results were obtained considering the patients' answers to the 20 items of the DACQ.

The mean level of awareness (normalized DACQ total score) was $69(\mathrm{SD}=11, \mathrm{~N}=367$; Figure 1). No significant differences in DACQ total or domain scores were found between younger and older patients or between males and females (Tables S5 and $\underline{\mathrm{S} 6})$. On the contrary, significant differences were identified among COPD severity groups ${ }^{5}$ in terms of disease acceptance $(P<0.0001)$, disease knowledge $(P=0.0007)$, and DACQ total score $(P=0.0084$; see Table S7 and Figure 2). Notably, significant differences were also found between patients with CAT $\geq 10$ vs CAT $<10$ for disease acceptance $(P<0.0001)$, awareness of treatment needs $(P=0.0453)$, disease knowledge $(P=0.0005)$, and DACQ total score $(P=0.0004$; see Table $\mathrm{S} 8$ and Figure 3).

The association between patients' awareness and their main demographic and clinical characteristics was investigated (Table 3). Consistent with the above-reported results, the CAT score negatively affected disease acceptance ( $\beta=-1.2944, P<0.0001$ ), awareness of treatment needs $(\beta=-0.4539, P=0.0003)$, and DACQ total score $(\beta=-0.4242$, $P<0.0001)$. However, there was no association with disease knowledge.

\section{Discussion}

The extent of patients' COPD awareness has been poorly investigated thus far. Despite the ongoing gainful efforts to increase COPD awareness, ${ }^{8}$ further actions are definitely needed to evaluate all domains involved and to launch appropriately tailored interventions.

During the SAT observational study, the DACQ was generated and tested following well-established procedures. The DACQ provides both a total score (indicating the global level of awareness) and specific scores about: 1) disease knowledge (the mastery of a correct knowledge on COPD characteristics), 2) awareness of treatment needs (the level of subjective view on necessity and role of COPD therapy), 3 ) disease perception (how the patient perceives COPD in terms of features and consequences), and 4) disease acceptance (the individual acceptance of thoughts and feelings related to $\mathrm{COPD}$ ).

DACQ proved to be easily understandable by patients and showed good reliability in terms of internal consistency, adequate construct validity, and moderate-to-good stability over time, making it suitable for the application. In the absence of a "gold standard" to evaluate awareness, we assessed the level of convergence of DACQ with the PROs. Convergent validity is generally considered adequate if the correlations with an instrument measuring the same construct is $>0.50 .{ }^{40}$ However, as recently proposed, ${ }^{41}$ if comparator constructs are only vaguely related to the construct under study, more flexibility in interpreting the correlation magnitude is allowed. The TSQM-9 and B-IPQ (evaluating treatment satisfaction 

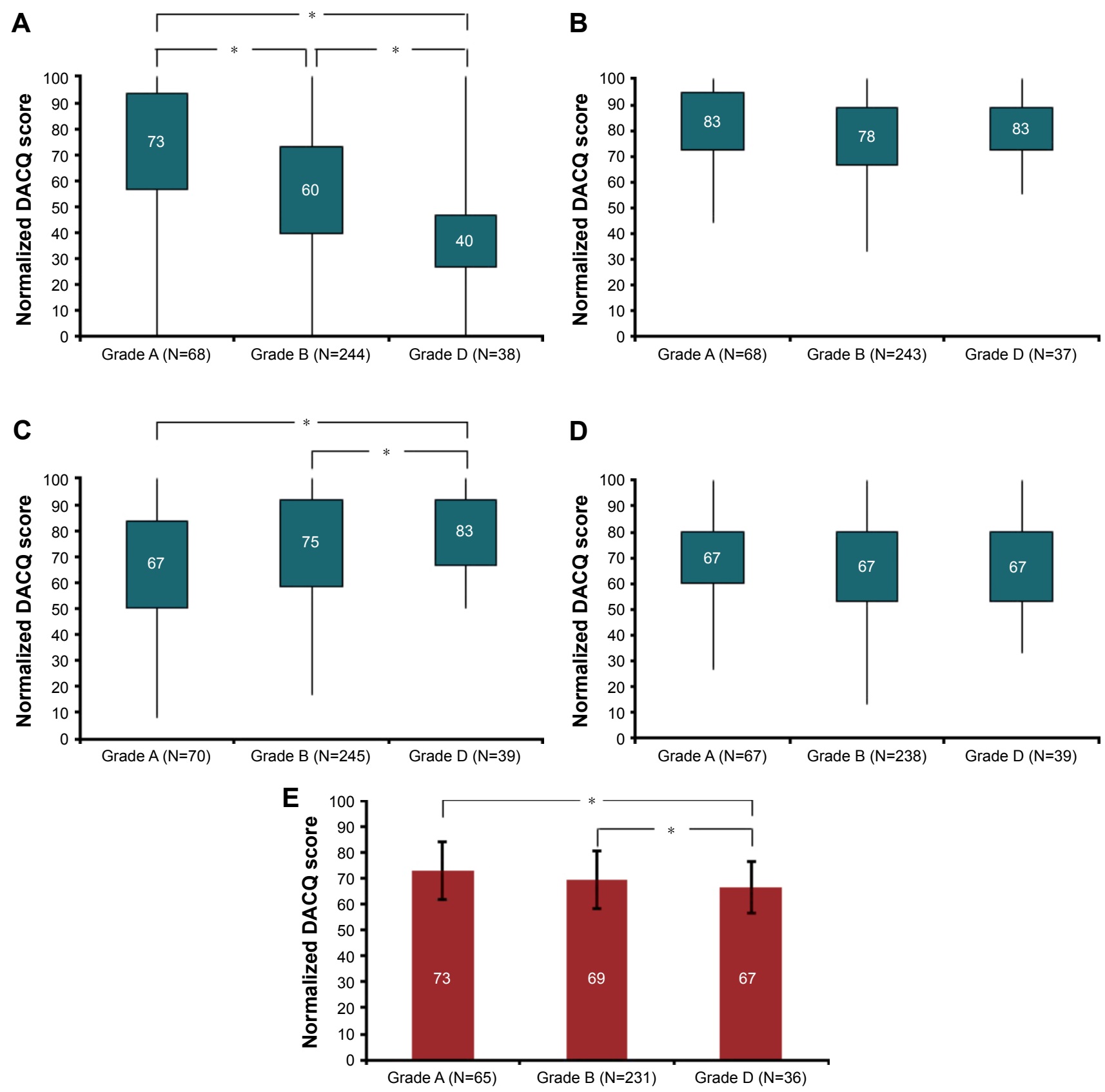

Figure 2 Patients' COPD awareness according to GOLD 2017 disease severity grades.

Notes: Normalized DACQ scores (range 0-100) for: (A) disease acceptance, (B) awareness of treatment needs, (C) disease knowledge, and (D) disease perception; median values (numbers), bottom and top of each box are the 25th and the 75th percentiles, respectively; bottom end and top end of the whiskers are minimum and maximum of all data, respectively. (E) Normalized DACQ total score; mean values (bars), I SD (whiskers). Higher scores represent higher levels of awareness. Asterisk (*) indicates a statistically significant difference $(P<0.05)$; the number of patients $(N)$ in each GOLD 2017 grade group is indicated. Only two patients were classified as GOLD 2017 grade; therefore, they were excluded from the analysis because of the insufficient sample size.

Abbreviation: DACQ, Disease Awareness in COPD Questionnaire.

and threatening views of diseases, respectively) were moderately correlated with awareness (DACQ total score), since they do not assess the same construct of DACQ. The correlation with CAT and mMRC was even lower, confirming the divergent validity. Good reliability in terms of internal consistency was achieved for all the domains, except for disease perception, which showed a Cronbach's $\alpha$ slightly below the acceptable threshold. This is probably due to the fact that the "disease perception" domain comprised the items showing lower factor loadings in the factor analysis. However, we decided not to remove them because we believed that they reinforce the overall assessment of the DAC construct.

In the patients referring to pulmonology centers and enrolled in the SAT study, the level of COPD awareness was 

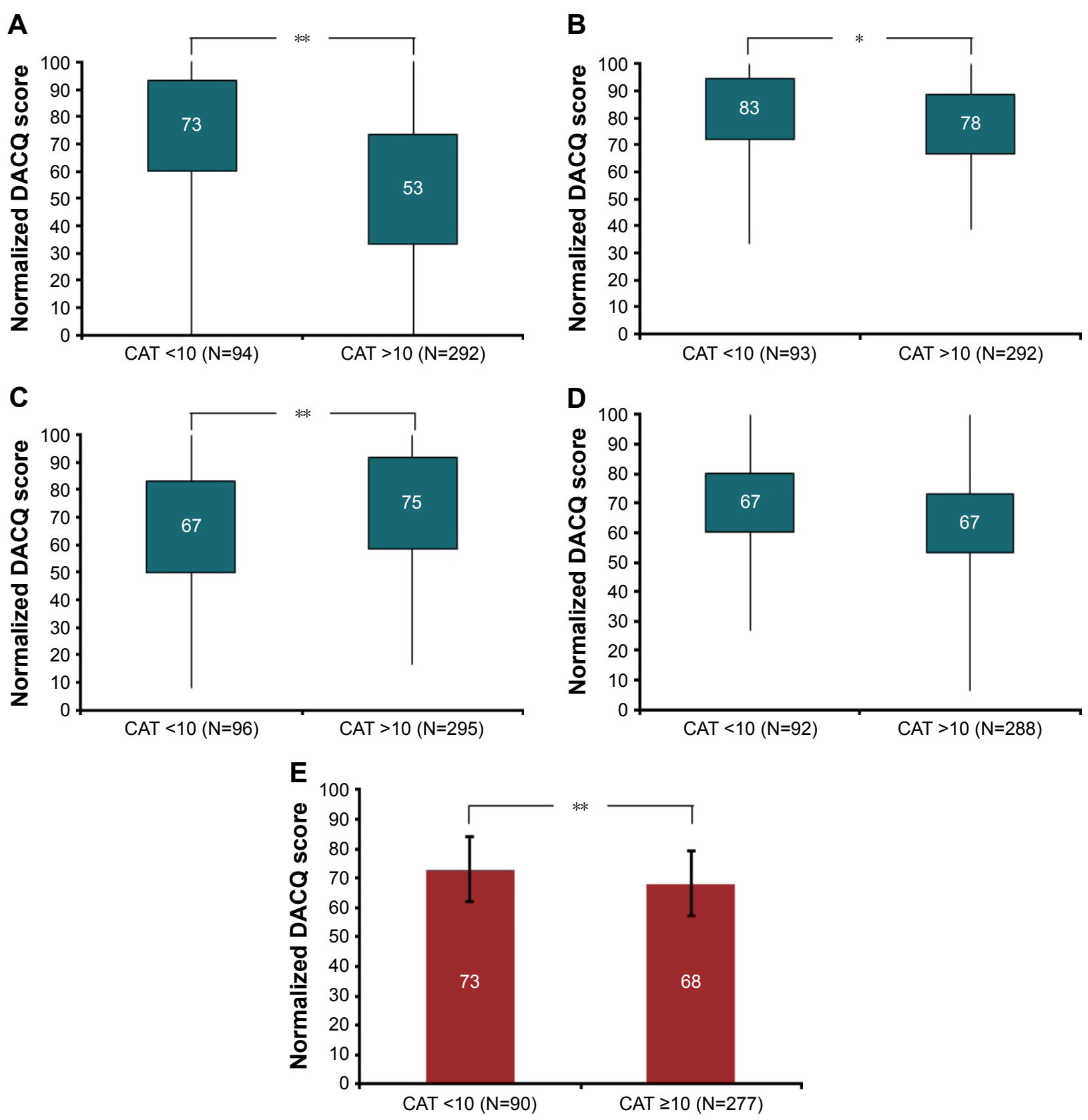

Figure 3 Patients' COPD awareness according to baseline CAT score.

Notes: Normalized DACQ scores (range 0-100) for: (A) disease acceptance, (B) awareness of treatment needs, (C) disease knowledge, and (D) disease perception; median values (numbers), bottom and top of each box are the 25th and the 75th percentiles, respectively; bottom end and top end of the whiskers are minimum and maximum of all data, respectively. (E) Normalized DACQ total score; mean values (bars), I SD (whiskers). Higher scores represent higher levels of awareness. Statistically significant differences: ${ }^{*} P<0.05$; $* * P<0.01$; the number of patients $(N)$ in each CAT group is indicated.

Abbreviations: CAT, COPD Assessment Test; DACQ, Disease Awareness in COPD Questionnaire.

suboptimal. Disease acceptance was particularly poor on average, while awareness of treatment needs and disease knowledge were higher. COPD awareness was not associated with either patients' age or gender. Disease awareness was lower in patients with a higher extent of symptoms in daily life. However, patients with a higher CAT score also had lower acceptance and consciousness of treatment needs, both of which are key components of awareness. This may explain why they had a limited global awareness compared with patients with lower CAT score. Therefore, the higher is the impact of symptoms, the lower is the acceptance of the disease and the greater necessity of therapy. This aspect could be due to patients' doubts about treatment efficacy since the pharmacological treatments for COPD neither cure nor provide immediate relief.

Patients with more severe COPD (GOLD grade D) are more likely to experience worsening COPD. This implies 
Table 3 Multivariable linear regression analysis: association between COPD awareness and baseline demographic/clinical characteristics of the patients

\begin{tabular}{|c|c|c|c|c|c|}
\hline & Age & $\begin{array}{l}\text { Gender } \\
\text { (female vs male) }\end{array}$ & CAT score & $\begin{array}{l}\text { COPD severity } \\
\text { (grade B vs grade A) }\end{array}$ & $\begin{array}{l}\text { COPD severity } \\
\text { (grade D vs grade A) }\end{array}$ \\
\hline Disease acceptance & $\begin{array}{l}\beta=0.1062 \\
P=0.5402\end{array}$ & $\begin{array}{l}\beta=-1.0278 \\
P=0.7269\end{array}$ & $\begin{array}{l}\beta=-1.2944 \\
P<0.000 I\end{array}$ & $\begin{array}{l}\beta=-0.1942 \\
P=0.9613\end{array}$ & $\begin{array}{l}\beta=-|1 .| 58 \mid \\
P=0.060 \mid\end{array}$ \\
\hline $\begin{array}{l}\text { Awareness of } \\
\text { treatment needs }\end{array}$ & $\begin{array}{l}\beta=-0.0077 \\
P=0.9383\end{array}$ & $\begin{array}{l}\beta=1.8417 \\
P=0.2800\end{array}$ & $\begin{array}{l}\beta=-0.4539 \\
P=0.0003\end{array}$ & $\begin{array}{l}\beta=2.5 I 84 \\
P=0.2774\end{array}$ & $\begin{array}{l}\beta=6.5718 \\
P=0.0555\end{array}$ \\
\hline Disease knowledge & $\begin{array}{l}\beta=-0.0939 \\
P=0.5020\end{array}$ & $\begin{array}{l}\beta=1.1211 \\
P=0.6378\end{array}$ & $\begin{array}{l}\beta=0.180 \mid \\
P=0.2988\end{array}$ & $\begin{array}{l}\beta=6.2181 \\
P=0.0533\end{array}$ & $\begin{array}{l}\beta=10.2216 \\
P=0.0316\end{array}$ \\
\hline Disease perception & $\begin{array}{l}\beta=-0.0228 \\
P=0.8654\end{array}$ & $\begin{array}{l}\beta=0.7215 \\
P=0.7499\end{array}$ & $\begin{array}{l}\beta=-0.0170 \\
P=0.9186\end{array}$ & $\begin{array}{l}\beta=-1.7595 \\
P=0.5709\end{array}$ & $\begin{array}{l}\beta=-0.2070 \\
P=0.9635\end{array}$ \\
\hline DACQ total score & $\begin{array}{l}\beta=-0.0017 \\
P=0.9837\end{array}$ & $\begin{array}{l}\beta=0.5367 \\
P=0.6986\end{array}$ & $\begin{array}{l}\beta=-0.4242 \\
P<0.0001\end{array}$ & $\begin{array}{l}\beta=0.5152 \\
P=0.7850\end{array}$ & $\begin{array}{l}\beta=0.0712 \\
P=0.9795\end{array}$ \\
\hline
\end{tabular}

Notes: Only two patients were classified as GOLD 2017 C grade; therefore, they were excluded from the analysis because of the limited group size. A higher CAT score represents a greater impact of symptoms in patient's daily life. Statistically significant results are highlighted in bold.

Abbreviations: CAT, COPD Assessment Test; DACQ, Disease Awareness in COPD Questionnaire.

that such patients should be more aware of their chronic condition and their need for life-lasting COPD treatments. Consistently, we found that disease knowledge was positively associated with COPD severity. In particular, grade D patients showed stronger background knowledge of their condition compared with grade A patients. Hence, the more severe the disease, the more informed are the patients. The possibility of evaluating the different components of awareness allows for the detection of the specific needs of each group of patients and for planning personalized strategies and interventions.

These study findings highlight that the patients' knowledge about COPD is quite satisfactory, while their acceptance and perception of the disease need to be enhanced. Targeted education programs, as well as a more effective communication between health care providers and patients, are desirable to improve patients' understanding and attitude to cope with their condition.

Our study was conducted in a real-life setting using the COPD severity staging according to GOLD 2017 recommendations, which is based on symptoms and history of exacerbations exclusively in which a large variability of severity staging at the individual patient level is proved. ${ }^{42}$ Most patients were classified as grade B (ie, with a lower risk of exacerbations but with a higher impact of COPD on their lives). Grade A, C, and D patients accounted for only $\sim 31 \%$ of the sample. Similarly, subjects with CAT $<10$ (ie, lower impact of symptoms) were approximately onefourth of the sample. However, in this observational study, no selection methods were implemented to examine more balanced groups of patients according to the investigated clinical characteristics because our aim was to survey a representative sample of the patients managed in the real-life specialist respiratory setting. Nevertheless, further studies in other settings, and with a different distribution of patients' severity and symptoms, would be useful to further explore our study findings.

Previous studies developed different kinds of surveys somehow related to awareness in patients with COPD by means of tools that did not undergo a proper validation process. A population-based questionnaire with $85 \%$ sensitivity and $45 \%$ specificity has been proposed as a screening tool for persons unaware of risk factors for COPD. ${ }^{34}$ An Internet-based survey focused on the perception of the disease and its exacerbations in a real-world population of patients with COPD, resulting in most patients being worried about long-term health and premature death. ${ }^{35} \mathrm{~A}$ national public survey with a structured questionnaire evaluated the perception of a large Italian population with obstructive lung diseases, highlighting the need for interventions to improve the awareness of these disorders and of COPD in particular. ${ }^{15}$ In the clinical setting, an Indian survey ${ }^{36}$ and a Korean study ${ }^{10}$ used different COPD awareness questionnaires and obtained similar results, indicating the urgent need for educating the public to increase the awareness of patient with COPD for the improvement of their self-management and quality of life.

Beyond these interesting results, the DACQ tool presented here is unique because it analyses different domains composing COPD awareness that may allow the patients to reach both knowledge and acceptance of the disease and its treatment. Therefore, the results of this study indicate that DACQ may represent a valid tool for further insights on DACQ.

\section{Conclusion}

The validated DACQ proved to be a reliable PRO instrument for measuring a complex, multifaceted construct such as 
disease awareness of COPD patients and it is easy to complete and score. This tool was developed during the SAT observational study in a real-life clinical setting. The results of this study showed an incomplete awareness of patients cared for in a specialist setting and provided the basic data for developing strategies to improve the diseases awareness in COPD patients.

\section{Acknowledgments}

Medical writing assistance was provided by Luisa Granziero, appointed by MediNeos Observational Research, Modena, Italy. Luisa Granziero declares no potential competing interests related to her assistance. We acknowledge the participants of the SAT study group for collecting data. This study was supported by Boehringer Ingelheim S.p.A, Italy. The SAT study group: Steering Committee: Fulvio Braido, A.O.U. IRCCS San Martino (Genova); Marco Contoli, A.O.U. Sant'Anna (Cona); Angelo Guido Corsico, Fondazione IRCCS San Matteo (Pavia); Fabiano Di Marco, Ospedale San Paolo (Milano); Paola Rogliani, Policlinico Tor Vergata (Roma); Pierachille Santus, Istituti Clinici Scientifici Maugeri-SpA SB (Milano); Nicola Scichilone, A.O. V. Cervello (Palermo). Participating centers: Rita Raccanelli, Dejan Radovanovic, Istituti Clinici Scientifici Maugeri-SpA SB (Milano); Angelo Guido Corsico, Erica Gini, Fondazione IRCCS San Matteo (Pavia); Vincenzo Patella, Giovanni Florio, Ospedale S. Maria della Speranza (Battipaglia); Fulvio Braido, Silvia Garuti, A.O.U. IRCCS San Martino (Genova); Marco Contoli, Giacomo Forini, A.O.U. Sant'Anna (Cona); Nicola Scichilone, Alida Benfante, A.O. V. Cervello (Palermo); Giuseppe Fiorentino, Antonella Marotta, A.O. dei Colli P.O. Monaldi (Napoli); Fabiano Di Marco, Fausta Alfano, Ospedale San Paolo (Milano); Paola Rogliani, Francesco Cavalli, Policlinico Tor Vergata (Roma); Roberto Piro, Patrizia Ruggiero, Arcispedale S. Maria Nuova IRCCS (Reggio Emilia); Mauro Carone, Maria Aliani, ICS Maugeri (IRCCS Cassano Murge); Antonio Iannaccone, Alessandro Izzo, A.O. San G. Moscati (Avellino); Biago Polla, Ospedale SS Antonio Biagio e Cesare e Arrigo (Alessandria); Riccardo Sarzani, Francesco Spannella, Ospedale INRCA (Ancona); Claudio Micheletto, Ospedale Mater Salutis (Legnago); Rigoletta Vincenti, Ospedale Civico (Livorno); Laura Maugeri, Carlo Gulotta - A.O.U. San Luigi Gonzaga (Orbassano); Roberto Tazza, Azienda Unità Sanitaria Locale Umbria n.2 (Terni); Luigi Di Re, Paolo Mimotti, Ospedale Mazzini (Teramo); Roberto Carbone, Rodolfo Riva, Ospedale Reg. Parini (Aosta). Project management, statistical analyses, and data management: Giovanni Fiori, Stefano Viaggi, Alessandra Ori, Lucia Simoni, Christian
Amici, Fabio Ferri, Barbara Roncari, Saide Sala, Francesca Trevisan, Nicole Lanci (MediNeos Observational Research, Modena, Italy).

\section{Author contributions}

All authors contributed to data analysis, drafting or revising the article, gave final approval of the version to be published, and agree to be accountable for all aspects of the work.

\section{Disclosure}

The following authors report personal fees for scientific consultation from Boehringer Ingelheim related to the study: FB, IB, AGC, MC, FDM, GM, PR, NS, and PS. FB has received honoraria for lectures at national and international meetings from Almirall, AstraZeneca, Boehringer Ingelheim, Chiesi Farmaceutici, Dompè, Guidotti/Malesci, GlaxoSmithKline, Menarini, Novartis, Lallemand Pharma, Biofutura, Levante Pharma, Merck Sharp and Dohme, and Zambon. He has served as consultant for AstraZeneca, Chiesi Farmaceutici, Novartis, Glaxo Smith Kline, Boehringer Ingelheim, Guidotti/ MalesciZambon, and CSL Behring. MC reports grants for research from AstraZeneca and Chiesi and reports personal fees for scientific consultations and/or lectures at national and international meetings from Chiesi, Novartis, AstraZeneca, Glaxo Smith Kline, Boehringer Ingelheim, Menarini, and Zambon, which are not related to the submitted manuscript. FDM has received honoraria for lectures at national and international meetings from Almirall, AstraZeneca, Boehringer Ingelheim, Chiesi Farmaceutici, Dompe, Guidotti/Malesci, GlaxoSmithKline, Menarini, Novartis, and Zambon. He has served as consultant for AstraZeneca, Chiesi Farmaceutici, Novartis, and Zambon, and he has received financial support for research from Novartis and Boehringer Ingelheim. PR has participated as a lecturer, speaker, and advisor in scientific meetings and courses under the sponsorship of Almirall, AstraZeneca, Biofutura, Boehringer Ingelheim, Chiesi, GlaxoSmithKline, Menarini Group, Mundipharma, and Novartis. Her department has received funding from Almirall, Boehringer Ingelheim, Chiesi, Novartis, and Zambon. She has no other relevant affiliations or financial involvement with any organization or entity with a financial interest in or financial conflict with the subject matter or materials discussed in the manuscript apart from those disclosed. PL and CS are employees of Boehringer Ingelheim Italy. FF is an employee of MediNeos Observational Research, Modena, Italy. He received payment from Boehringer Ingelheim Italy for the following activities related to the submitted work: scientific support, clinical operations, data management, statistical analysis, and manuscript preparation. $\mathrm{MC}, \mathrm{GF}$, and 
VP do not report any competing interests. The authors report no other conflicts of interest in this work.

\section{References}

1. Rennard SI, Drummond MB. Early chronic obstructive pulmonary disease: definition, assessment, and prevention. Lancet. 2015;385(9979): $1778-1788$.

2. Martinez CH, Richardson CR, Han MK, Cigolle CT. Chronic obstructive pulmonary disease, cognitive impairment, and development of disability: the health and retirement study. Ann Am Thorac Soc. 2014;11(9): 1362-1370.

3. Braido F, Baiardini I, Menoni S, et al. Disability in COPD and its relationship to clinical and patient-reported outcomes. Curr Med Res Opin. 2011;27(5):981-986.

4. Corli O, Apolone G, Pizzuto M, et al. Illness awareness in terminal cancer patients: an Italian study. Palliat Med. 2009;23(4):354-359.

5. GOLD. Global Initiative for Chronic Obstructive Lung Disease [webpage on the Internet]. Global strategy for the diagnosis, management, and prevention of chronic obstructive pulmonary disease (updated 2017); 2016. Available from: www.goldcopd.or. Accessed December 2016.

6. Zielinski J, Bednarek M, Górecka D, et al. Increasing COPD awareness. Eur Respir J. 2006;27(4):833-852.

7. NIH National Heart Lung and Blood Institute [webpage on the Internet]. National asthma control initiative; 2018. Available from: www.nhlbi.gov. Accessed February 6, 2018.

8. NIH National Heart Lung and Blood Institute [webpage on the Internet]. COPD learn more breathe better ${ }^{\mathbb{B}}$ program; 2018 . Available from: https://www.nhlbi.nih.gov/health/educational. Accessed last accessed February 6, 2018.

9. Hernandez P, Balter M, Bourbeau J, Hodder R. Living with chronic obstructive pulmonary disease: a survey of patients' knowledge and attitudes. Respir Med. 2009;103(7):1004-1012.

10. Seo JY, Hwang YI, Mun SY, et al. Awareness of COPD in a high risk Korean population. Yonsei Med J. 2015;56(2):362-367.

11. World Health Organization [webpage on the Internet]. Adherence to long-term therapies: evidence for action; 2003. Available from: www. who.int/chp/knowledge/publications/adherence_report/en/index.html. Accessed February 7, 2018.

12. Huetsch JC, Uman JE, Udris EM, Au DH. Predictors of adherence to inhaled medications among Veterans with COPD. J Gen Intern Med. 2012;27(11):1506-1512.

13. Krauskopf K, Federman AD, Kale MS, et al. Chronic obstructive pulmonary disease illness and medication beliefs are associated with medication adherence. COPD. 2015;12(2):151-164.

14. Mäkelä MJ, Backer V, Hedegaard M, Larsson K. Adherence to inhaled therapies, health outcomes and costs in patients with asthma and COPD. Respir Med. 2013;107(10):1481-1490.

15. Braido F, Baiardini I, Sumberesi M, Blasi F, Canonica GW. Obstructive lung diseases and inhaler treatment: results from a national public pragmatic survey. Respir Res. 2013;14:94.

16. Broadbent E, Petrie KJ, Main J, Weinman J. The brief illness perception questionnaire. J Psychosom Res. 2006;60(6):631-637.

17. Jones PW, Harding G, Berry P, Wiklund I, Chen WH, Kline Leidy N. Development and first validation of the COPD Assessment Test. Eur Respir J. 2009;34(3):648-654.

18. Atkinson MJ, Kumar R, Cappelleri JC, Hass SL. Hierarchical construct validity of the treatment satisfaction questionnaire for medication (TSQM version II) among outpatient pharmacy consumers. Value Health. 2005;8(Suppl 1):S9-S24.

19. Atkinson MJ, Sinha A, Hass SL, et al. Validation of a general measure of treatment satisfaction, the Treatment Satisfaction Questionnaire for Medication (TSQM), using a national panel study of chronic disease. Health Qual Life Outcomes. 2004;2:12.
20. Munari AB, Gulart AA, Dos Santos K, Venâncio RS, Karloh M, Mayer AF. Modified Medical Research Council Dyspnea Scale in GOLD Classification Better Reflects Physical Activities of Daily Living. Respir Care. 2018;63(1):77-85.

21. Benhamou M, Baron G, Dalichampt M, et al. Development and validation of a questionnaire assessing fears and beliefs of patients with knee osteoarthritis: the Knee Osteoarthritis Fears and Beliefs Questionnaire (KOFBeQ). PLoS One. 2013;8(1):e53886.

22. Andrich D. Rasch Models for Measurement. Beverly Hills, CA: SAGE Publications Inc; 1988.

23. Rasch G. Probabilistic models for some intelligence and attainment tests. Expanded ed. Chicago, IL: University of Chicago Press; 1980.

24. van der Linden W, Hambleton R. Handbook of Modern Item Response Theory. New York, NY: Springer Science + Business Media; 1997.

25. Wright BD, Linacre JM. Reasonable mean-square fit values. Rasch Meas Trans. 1994;8:370.

26. Wu M, Adams R. Applying the Rasch Model to Psycho-Social Measurement. A Practical Approach. Melbourne, Australia: Educational Measurement Solutions; 2007.

27. Baiardini I, Braido F, Molinengo G, et al. Chronic Urticaria Patient Perspective (CUPP): the first validated tool for assessing quality of life in clinical practice. J Allergy Clin Immunol Pract. 2018;6(1):208-218.

28. Molinengo G, Baiardini I, Braido F, Loera B. RhinAsthma patient perspective: a Rasch validation study. J Asthma. 2018;55(2):119-123.

29. Cronbach LJ. Coefficient alpha and the internal structure of tests. Psychometrika. 1951;16(3):297-334.

30. Nunnaly J, Bernstein IH. Psychometric Theory. 3rd ed. New York, NY: McGraw-Hill; 1994.

31. Koo TK, Li MY. A guideline of selecting and reporting intraclass correlation coefficients for reliability research. JChiropr Med. 2016;15(2): $155-163$.

32. Schafer JL. Multiple imputation: a primer. Stat Methods Med Res. 1999;8(1):3-15.

33. Post MW. What to do with "moderate" reliability and validity coefficients? Arch Phys Med Rehabil. 2016;97(7):1051-1052.

34. Calverley PM, Nordyke RJ, Halbert RJ, Isonaka S, Nonikov D. Development of a population-based screening questionnaire for COPD. COPD. 2005;2(2):225-232.

35. Barnes N, Calverley PM, Kaplan A, Rabe KF. Chronic obstructive pulmonary disease and exacerbations: patient insights from the global Hidden Depths of COPD survey. BMC Pulm Med. 2013;13:54.

36. Thakrar R, Alaparthi GK, Kumar SK, Vaishali K, Zulfeequer CP, Aanad R. Awareness in patients with COPD about the disease and pulmonary rehabilitation: a survey. Lung India. 2014;31(2):134-138.

37. Soriano JB, Calle M, Montemayor T, Alvarez-Sala JL, Ruiz-Manzano J, Miravitlles M. The general public's knowledge of chronic obstructive pulmonary disease and its determinants: current situation and recent changes. Arch Bronconeumol. 2012;48(9):308-315.

38. Mun SY, Hwang YI, Kim JH, et al. Awareness of chronic obstructive pulmonary disease in current smokers: a nationwide survey. Korean $J$ Intern Med. 2015;30(2):191-197.

39. Edwards JR. Multidimensional Constructs in Organizational Behavior Research: An Integrative Analytical Framework, Organizational Research Methods. Vol 4 No 2. Thousand Oaks, CA: SAGE Publications, Inc; 2001:144-192.

40. Terwee CB, Bot SD, de Boer MR, et al. Quality criteria were proposed for measurement properties of health status questionnaires. $J$ Clin Epidemiol. 2007;60:34-42.

41. Abma IL, Rovers M, van der Wees PJ. Appraising convergent validity of patient-reported outcome measures in systematic reviews: constructing hypotheses and interpreting outcomes. BMC Res Notes. 2016;9:226.

42. Soriano JB, Hahsler M, Soriano C, et al. Temporal transitions in COPD severity stages within the GOLD 2017 classification system. Respir Med. 2018;142:81-85. 
International Journal of COPD

\section{Publish your work in this journal}

The International Journal of COPD is an international, peer-reviewed journal of therapeutics and pharmacology focusing on concise rapid reporting of clinical studies and reviews in COPD. Special focus is given to the pathophysiological processes underlying the disease, intervention programs, patient focused education, and self management protocols

This journal is indexed on PubMed Central, MedLine and CAS. The manuscript management system is completely online and includes a very quick and fair peer-review system, which is all easy to use. Visit http://www.dovepress.com/testimonials.php to read real quotes from published authors 